\title{
Rotation Curve and Metallicity of the Outer Disc from Cepheids
}

\author{
F. Pont, D. Queloz, M. Mayor and P. Bratschi \\ Geneva Observatory, Sauverny, Switzerland
}

\begin{abstract}
A group of faint, remote Cepheids in the outer disc of the Galaxy has been observed in spectroscopy and photometry. These Cepheids are used as tracers to measure the rotation curve of the disc beyond $\mathrm{R}=11 \mathrm{kpc}$, and to study the metallicity gradient in the outer disc.
\end{abstract}

\section{Rotation curve of the outer disc from Cepheids}

Cepheids have been used to estimate the rotation curve of the Galaxy since Joy (1939), and the present sample of Cepheids measured in radial velocity determines the rotation curve for galactocentric radii $\mathrm{R}$ between 6 and $11.5 \mathrm{kpc}$ (Caldwell \& Coulson 1987, Pont et al. 1994). At larger radii, our knowledge comes mainly from HII regions, which are not very accurate distance indicators and show an ambiguous, ill-defined rise of the rotation curve beyond $11 \mathrm{kpc}$.

We have been measuring since 1993 radial velocities for a group of faint $\left(\mathrm{m}_{v}=11-15 \mathrm{mag}\right)$, distant $(\mathrm{R}>11 \mathrm{kpc})$ Cepheids of the outer disc, with the aim of obtaining a precise determination of the rotation curve up to $R=16 \mathrm{kpc}$.

Preliminary results were presented in Pont et al. (1995) and outline a rotation curve significantly lower than that given by HII regions. This can mean either a distance scale difference between Cepheids and HII regions, or a real difference in their kinematics.

\section{Metal deficiency of outer disc Cepheids}

The spectra that we used to measure radial velocities allow us to address another question : the metal deficiency of outer disc Cepheids. A good knowledge of the metallicity gradient in the outer disc is necessary to correct Cepheid distances for metallicity effects; it is also an essential constraint on the chemical history of the Milky Way.

We collected for each Cepheid up to 8 high-resolution, low signal-to-noise ratio spectra distributed over the pulsation phase. The spectra were then crosscorrelated with a numerical template. The area of the cross-correlated function (CCF) is a measurement of the mean area of the spectral lines. It is therefore a sensitive function of temperature, metallicity, gravity and microturbulence (Queloz 1995).

In order to isolate the metallicity effect, we determine the influences of the other parameters by following Cepheids along their pulsating cycles (see Fig. 
1a). We do not yet have an absolute calibration of the CCF area in terms of $[\mathrm{Fe} / \mathrm{H}]$. However, the small scatter of the CCF area gradient as a function of R (Fig. 1b), especially for solar neighbourhood calibrators, indicates that this technique is full of promises. We shall complete the calibration soon and thus get good quality spectroscopic metallicity estimates for the faint Cepheids of our sample.

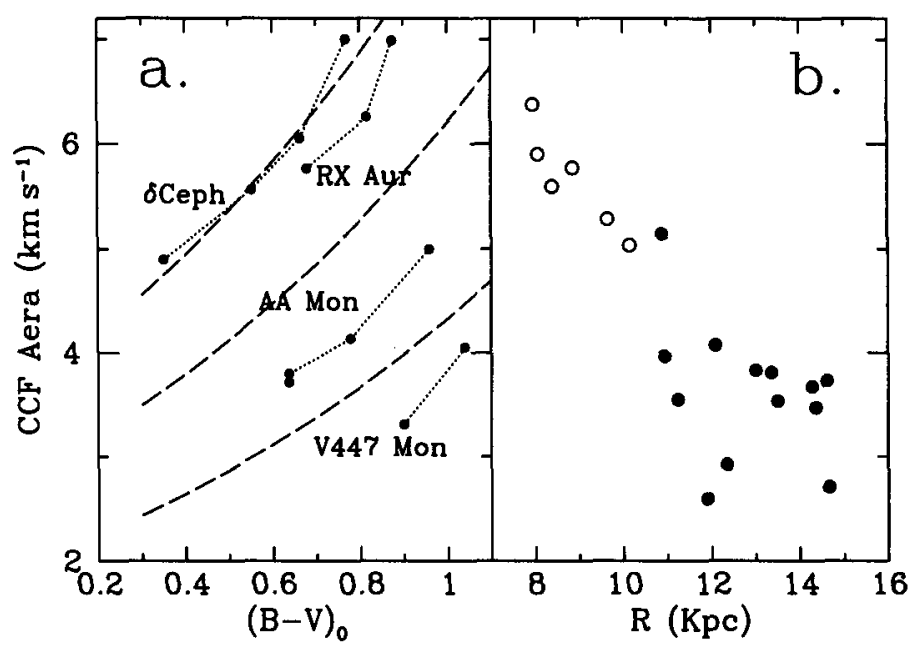

Figure 1. (a) Variation of the area of the CCF during the pulsation cycle for Cepheids of various metallicities, as a function of dereddened colour ( $\delta$ Cep, RX Aur: solar neighbourhood calibrators; V447 Mon, AA Mon: deficient outer disc Cepheid). The dashed lines represent loci of constant metallicity obtained by an analytical fit. Changes induced by microturbulence were removed, to the first order, by ignoring spectra taken when microturbulence is important, namely during the ascending part of the light curve. (b) Average CCF area for each Cepheid of our sample corrected from the temperature effect, as a function of distance form the galactic centre. The solar neighbourhood calibration Cepheids and the outer disc Cepheids are shown as open and filled circles respectively. The metallicity gradient in the disc appears clearly, showing the CCF area to be a very sensitive metallicity indicator.

\section{References}

Caldwell, J.A.R., \& Coulson, I. 1987, AJ, 93, 1090

Joy A. H. 1939, ApJ, 89, 356

Pont, F., Mayor, M., \& Burki, G. 1994, A\&A, 285, 415

Pont, F., et al. 1995, in: IAU Symp. 169, ed. L. Blitz, in press

Queloz, D. 1995, in: IAU Symp. 167, ed. A.G. Davis Philip, in press 